\title{
Efficacy of Continuously Administered PEDF-Derived Synthetic Peptides against Osteosarcoma Growth and Metastasis
}

\author{
Matthew L. Broadhead, ${ }^{1}$ Peter F. M. Choong, ${ }^{1,2}$ and Crispin R. Dass ${ }^{3}$ \\ ${ }^{1}$ Department of Orthopaedics and Department of Surgery, St. Vincent's Hospital, University of Melbourne, \\ Fitzroy VIC 3065, Australia \\ ${ }^{2}$ Sarcoma Service, Peter MacCallum Cancer Centre, East Melbourne, VIC 3002, Australia \\ ${ }^{3}$ School of Biomedical and Health Sciences, Victoria University, St. Albans, VIC 3021, Australia \\ Correspondence should be addressed to Matthew L. Broadhead, matthew.broadhead@gmail.com \\ Received 5 December 2011; Revised 18 February 2012; Accepted 19 February 2012 \\ Academic Editor: Susan E. Crawford
}

Copyright ( $) 2012$ Matthew L. Broadhead et al. This is an open access article distributed under the Creative Commons Attribution License, which permits unrestricted use, distribution, and reproduction in any medium, provided the original work is properly cited.

\begin{abstract}
The potent antiangiogenic pigment epithelium-derived factor (PEDF) has shown promise against osteosarcoma, a tumour that originates in the bone and metastasises to the lungs. Neurotrophic, antiangiogenic, antiproliferative, and antimetastatic properties of PEDF have been attributed to a number of functional epitopes on the PEDF glycoprotein. StVOrth-2 (residues 78-102) and StVOrth-3 (residues 90-114) are two PEDF-derived peptides based on these functional epitopes. StVOrth-2 has previously been shown to inhibit osteosarcoma cell proliferation, while StVOrth-3 increased osteosarcoma cell adhesion to collagen I in vitro. In this paper, we have evaluated systemically and continuously delivered StVOrth-2 and StVOrth-3 using a clinically relevant murine model of osteosarcoma with spontaneous metastasis. Treatment with StVOrth-2 or StVOrth-3 with microosmotic pumps was initiated after primary osteosarcoma was established in the tibia. While treatment with StVOrth-2 and StVOrth-3 did not appear to affect local tumour invasion, tumour necrosis or apoptosis, StVOrth-2 predominantly restricted the growth of primary tumours, while StVOrth-3 restricted the burden of pulmonary metastatic disease. No peptide caused gross toxicity in mouse tissues as assessed by measuring weight of animals, serum biochemistry, and gross tissue observation. The differential effects exhibited by StVOrth-2 and StVOrth-3 in this orthotopic model of osteosarcoma may be related to the functional epitopes on the PEDF glycoprotein that they represent.
\end{abstract}

\section{Introduction}

Pigment epithelium-derived factor (PEDF) is a $50 \mathrm{kDa}$ endogenous glycoprotein that was first discovered in 1991 as a factor secreted by the pigment epithelium of the human foetal eye [1]. PEDF was shown to promote differentiation of retinoblastoma cells [2] and was implicated in a range of eye pathologies including diabetic retinopathy, macular degeneration, and retinitis pigmentosa and glaucoma [3]. PEDF is a potent antiangiogenic agent, more potent than angiostatin, endostatin and thrombospondin-1 by endothelial cell migration assay [4]. PEDF has also been shown to be an antitumorigenic agent for malignancies including osteosarcoma, melanoma, glioma, lung, breast, prostatic, ovarian and pancreatic carcinomas [5].

Biochemical studies have enabled the identification of multiple functional epitopes for PEDF. The interactions between these epitopes and receptors are likely to initiate divergent signalling pathways for the different cellular effects of PEDF. Filleur et al. [6] first characterised 34-mer (residues 24-57) and 44-mer (residues 58-101) PEDF-derived peptides that conferred antiangiogenic and neurotrophic activity, respectively. Additionally, another sequence, ERT (residues 79-94), showed both antiangiogenic and differentiation activity. The properties of these peptides were demonstrated in vitro by endothelial cell apoptosis and chemotaxis assays and Y-79 retinoblastoma differentiation assay. Using a subcutaneous tumour model and PC3 prostate cancer cells, expression of the 34 mer peptide reduced tumour microvascular density and induced tumour cell apoptosis, effects not demonstrated for the 44-mer peptide [6]. The 34 mer peptide restricted angiogenesis through a c-jun-NH2 kinase (JNK-) dependent pathway leading to NFATc2 deactivation and c-Flip antagonism [6]. 
StVOrth-2 (residues 78-102)

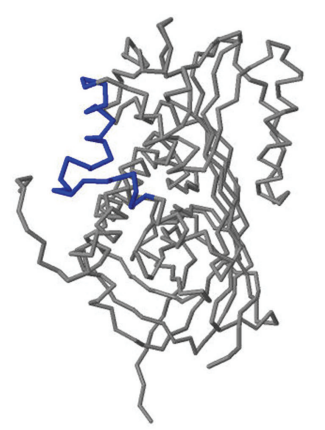

StVOrth-3 (residues 90-114)

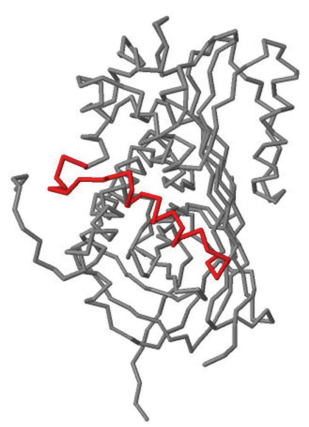

Inhibits OS cell proliferation
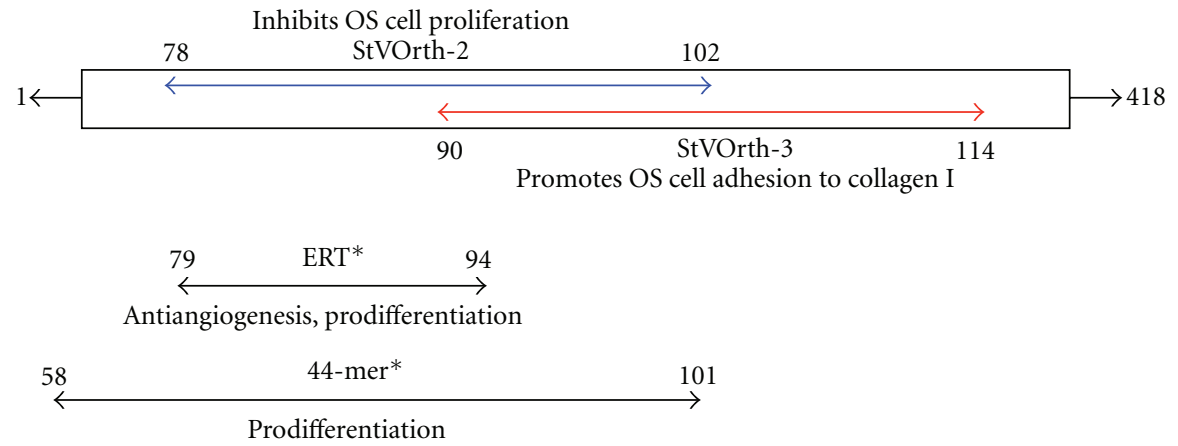

Prodifferentiation

FIgure 1: StVOrth-2 and StVOrth-3 peptides. StVOrth-2 (highlighted in blue) consists of residues 78-102 of the parent PEDF sequence. StVOrth-3 (highlighted in red) consists of residues 90-114. StVOrth-2 and StVOrth-3 have previously been shown to inhibit osteosarcoma cell proliferation and promote osteosarcoma cell adhesion to collagen I in vitro, respectively [7]. * ERT and 44-mer peptide sequences, as described by Filleur et al. [6], overlap with the StVOrth-2 and StVOrth-3 sequences.

In another study, four different PEDF-derived peptides, termed StVOrth-1, StVOrth-2, StVOrth-3, and StVOrth4, consisting of PEDF residues 40-64, 78-102, 90-114, and 387-411, respectively, were tested in vitro and in vivo [7]. In vitro, StVOrth-2 was the most potent inhibitor of SaOS-2 osteosarcoma cell proliferation, while StVOrth3 dramatically promoted SaOS-2 adhesion to collagen I. StVOrth-4 inhibited SaOS-2 cell invasion through Matrigel. StVOrth-1, -2, and -3 all induced osteoblastic differentiation. StVOrth-3 and StVOrth-4 reduced VEGF expression in SaOS-2 osteosarcoma cells. StVOrth-2 and StVOrth-3 were then evaluated in vivo using an orthotopic murine model of osteosarcoma. Notably, StVOrth-2 (residues 78-102) and StVOrth-3 (residues 90-114) possessed sequences that overlapped with the 44-mer (residues 58-101) and ERT (residues 79-94) sequences described by Filleur et al. [6] (Figure 1). Both StVOrth-2 and StVOrth-3 restricted osteosarcoma tumour growth and inhibited the development of pulmonary metastases when SaOS- 2 cells were treated prior to intratibial injection.

The findings of both Filleur et al. [6] and Ek et al. [7] provide some insight into how PEDF structure relates to its multidimensional ability to restrict tumour progression. However, the study design and methods of peptide delivery used in these models make it difficult to extrapolate the findings for human use. Filleur et al. [6] used a subcutaneous tumour model with transfected PC-3 cells to demonstrate the differential effects of the 34-mer and 44-mer PEDFderived peptides. Gene therapies have yet to be proven safe for human application, making it unlikely that they will be used for osteosarcoma therapy in the near future [8]. Ek et al. [7] used the SaOS-2 osteosarcoma cell line to achieve a spontaneously metastasizing murine model of orthotopic osteosarcoma. SaOS-2 cells were treated with StVOrth-2 and StVOrth-3 peptides prior to intraosseous injection, thus facilitating early uptake of peptides and change in phenotype. In order to evaluate the true efficacy of PEDFderived peptides against established osteosarcoma, treatment should be delayed until after the establishment of primary tumours. This would better simulate the clinical presentation and treatment of osteosarcoma in humans.

\section{Materials and Methods}

2.1. Cells and Culture Conditions. The SaOS-2 human osteosarcoma cell line (American Tissue Culture Collection, Manassas, VA, USA) was cultured in complete medium, $\mathrm{CM}$, at $37^{\circ} \mathrm{C}$ and in humidified $5 \% \mathrm{CO}_{2}$. CM consisted of MEM-Alpha+GlutaMAX (Invitrogen, Carlsbad, CA, USA) supplemented with $10 \%$ foetal bovine serum (Invitrogen, Carlsbad, CA, USA) and 1\% antibiotic-antimycotic (Invitrogen, Carlsbad, CA, USA). Exponentially growing cells, with passage number always less than 20 , were used for the studies. 
2.2. Establishment of the Orthotopic Model of Osteosarcoma. 5-week-old Balb/c nude mice were purchased from the Animal Resource Centre, Perth, Australia and were housed at the St Vincent's Hospital BioResources Centre under PC2 pathogen-free conditions. Animal ethics approval was obtained from the St Vincent's Hospital Melbourne Animal Ethics Committee. A 50\% concentration of Matrigel was used to dilute SaOS-2 osteosarcoma cells to a concentration of $2 \times 10^{6}$ cells $/ \mathrm{mL}$. Following anaesthesia with intraperitoneal ketamine $(100 \mathrm{mg} / \mathrm{kg})$ and xylazine $(10 \mathrm{mg} / \mathrm{kg})$, a 27 gauge needle was introduced into the left tibia of each mouse using a gentle drilling motion in order to avoid iatrogenic fracture [9], and a volume of $10 \mu \mathrm{L}$ of SaOS-2/Matrigel solution was injected. Postinjection, the needle was retracted slowly to prevent backflow of injectate.

Tumour growth and animal weights were monitored twice weekly until the endpoint of the study. Anteroposterior (AP) and lateral (L) dimensions of limbs were recorded using digital callipers. Volumes were calculated from these dimensions using the formula $4 / 3 \pi[1 / 4(\mathrm{AP}+\mathrm{L})]^{2}$ [7]. The contralateral nontumour-bearing limb was used as a control to calculate actual tumour volume.

Primary orthotopic tumours were apparent at day 20 after SaOS-2 inoculation, when average tumour volume was $22.5 \mathrm{~mm}^{3}$, at which point mice were randomised into treatment groups each consisting of four mice. As outlined below, these groups received either: (1) sterile water as control, (2) StVOrth-2 at $50 \mu \mathrm{g} / \mathrm{kg} /$ day, (3) StVOrth-2 at $500 \mu \mathrm{g} / \mathrm{kg} / \mathrm{day}$, (4) StVOrth-3 at $50 \mu \mathrm{g} / \mathrm{kg} / \mathrm{day}$, or (5) StVOrth-3 at $500 \mu \mathrm{g} / \mathrm{kg} / \mathrm{day}$.

2.3. Delivery of PEDF-Derived Synthetic Peptides. PEDFderived peptides, StVOrth-2 (residues 78-102) and StVOrth3 (90-114), were designed and sourced previously according to the procedure outlined by Ek et al. [7]. This paper refers to the full-length human PEDF sequence, and amino acid numbering is based on those sequences listed in GenBank (National Institutes of Health). StVOrth-2 is the aminoacid sequence ${ }_{78}$ VLLSP LSVAT ALSAL SLGAE QRTES 102 . StVOrth-3 is the sequence ${ }_{90}$ SALSL GAEQR TESII HRALY YDLIS $_{114}$. High-performance liquid chromatography (HPLC) and mass spectrometry was used to confirm the purity of these peptides. Ek et al. [7] showed that StVOrth-2 inhibited SaOS-2 osteosarcoma cell proliferation, while StVOrth-3 inhibited SaOS-2 osteosarcoma cell adhesion to collagen I in vitro. StVOrth-2 and StVOrth-3 sequences overlap with the 44-mer (residues 58-101), and ERT (residues 79-94) sequences described by Filleur et al. [6] (Figure 1).

Sustained delivery of StVOrth-2, StVOrth-3, or sterile water (negative control, placebo) was achieved by intraperitoneally implanted Alzet microosmotic pump (Durect Corp., Cupertino, CA, USA). Pumps were aseptically filled with the different treatments and surgically implanted within the peritoneal cavity of animals for systemic delivery. This surgery was performed at day 20 after SaOS-2 injection. The mean pumping rate for the Alzet microosmotic pump (model 1002) is $0.25 \mu \mathrm{L} / \mathrm{hr}$ over 14 days, as determined by the manufacturer. StVOrth-2 and StVOrth-3 were administered at $50 \mu \mathrm{g} / \mathrm{kg}$ and $500 \mu \mathrm{g} / \mathrm{kg}$ daily doses. Sterile water was used as diluent for StVOrth-2 and StVOrth-3.

The human physiological serum concentration of PEDF ranges between $4 \mathrm{ng} / \mathrm{mL}(80 \mathrm{pM})$ to $15 \mu \mathrm{g} / \mathrm{mL}(300 \mathrm{nM})$ [1015]. In one study, inhibition of vessel formation in ischemiainduced retinopathy was achieved at a $50 \mathrm{nM}$ concentration [16]. The $50 \mu \mathrm{g} / \mathrm{kg}$ and $500 \mu \mathrm{g} / \mathrm{kg}$ doses used in the present study are equivalent to $1 \mu \mathrm{g} / \mathrm{mL}(20 \mathrm{nM})$ and $10 \mu \mathrm{g} / \mathrm{mL}$ (200 nM) concentrations of PEDF, respectively, which lies within the range mentioned above. This assumes an average mouse weight of 20 grams and an average blood volume of $1 \mathrm{~mL}$ [17]. It is known that the pumps are capable of delivering a steady state quantity of peptides into the abdominal cavity $[18,19]$, which will eventually be taken into the microvasculature supplying the abdominal region.

2.4. Study Endpoint and Tissue Analysis. Tumours had grown to a disabling size for control animals at day 34 after SaOS2 inoculation as expected. This was the humane endpoint of the study, and all animals were euthanized under anaesthesia by cervical dislocation at this time. Following this, tumourbearing limbs, lungs, hearts, small intestines, and skin were harvested for examination. All specimens were fixed in $4 \%$ paraformaldehyde, followed by paraffin embedding. Blood samples were obtained after cervical dislocation and dissection through the thoracic cage and were immediately treated with anticoagulants. Affected limbs were X rayed at $35 \mathrm{kV}$ for $30 \mathrm{~s}$ using a cabinet system (Faxitron Corp., Wheeling, IL, USA).

Blood collected after euthanasia was analysed for renal and hepatic biochemical parameters (serum creatinine, alanine transaminase (ALT), and aspartate transferase (AST)) using a Sysmex XE2100 instrument [20]. Tissues were embedded in paraffin prior to histological preparation and analysis. For preparation of paraffin sections, $4 \mu \mathrm{m}$ sections of tumours and viscera were cut by microtome. Tumours were sectioned to provide an en face surface for the quantification of per cent tumour necrosis and apoptosis.

Primary tumours, lungs, heart, small intestine, and skin sections were dehydrated through an ethanol series followed by xylene, then stained with haematoxylin and eosin. A terminal dUTP nick end labelling (TUNEL) assay kit (Promega, Madison, WI, USA) was used to detect apoptotic cells in primary tumours [21]. Pertex mounting agent was used to seal coverslips to slide sections, and all tissues were observed using a Nikon Eclipse TE2000-U microscope (Nikon, Lidcombe, NSW, Australia) and photographed with SPOT Advanced software (SciTech, Aurora, IL, USA).

The effect of intraperitoneal StVOrth-2 and StVOrth-3 on the development of pulmonary metastatic disease was examined histologically. Lungs were sectioned to achieve the greatest cross-sectional area and stained with haematoxylin and eosin. Micrometastases were identified by systematically scanning lung sections under 20x magnification. Discernible clusters of metastatic cells at this magnification were counted as micrometastases. Ten metastatic lesions were then randomly selected from each treatment group for measurement of cross-sectional areas. 

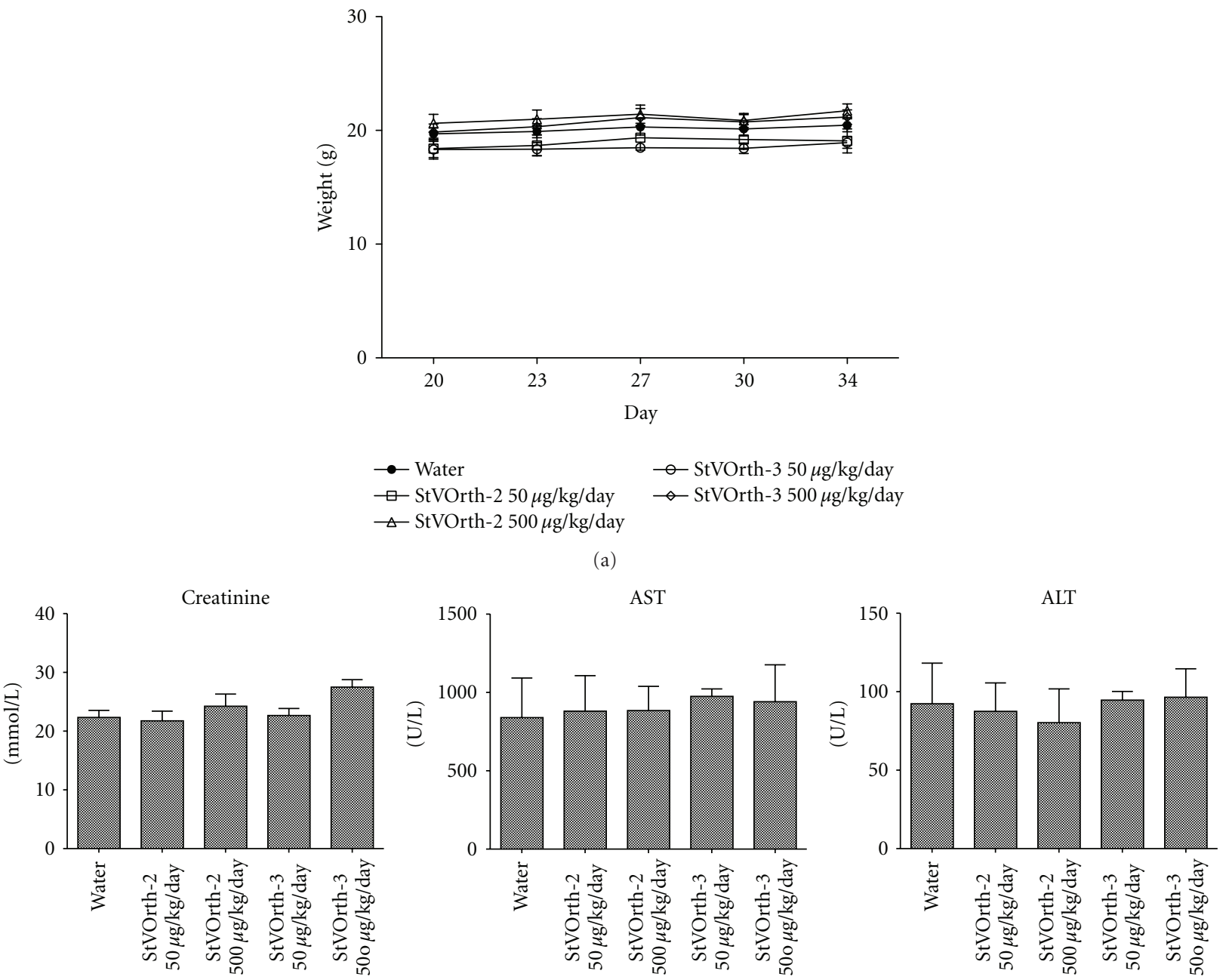

(b)

FIgURE 2: (a) Animal weights recorded at days 20, 23, 27, 30, and 34 after SaOS-2 intratibial injection. There was no significant weight loss compared to control. (b) Biochemical analysis of serum collected postmortem ( \pm SEM). There was no evidence of renal or hepatic toxicity.

2.5. Statistical Methods and Imaging Software. One-way and two-way ANOVA analyses with Bonferroni multiple comparisons test were used where appropriate (GraphPad Prism 5 for Mac OS X). ImageJ (Version 1.45j, National Institutes of Health, USA) was used for all image analysis. Means and standard errors were calculated for all data collected.

\section{Results}

3.1. Potential Systemic Side Effects of PEDF-Derived Peptides. Mice were studied for potential systemic side affects associated with delivery of StVOrth-2 (residues 78-102) and StVOrth-3 (residues 90-114) (see Figure 1). Mice were regularly monitored during the study for signs of distress and animal weights were recorded twice weekly. All mice remained well for the duration of the study, and no significant weight loss compared to control was observed (two-way ANOVA) (Figure 2(a)).

Serum, hearts, lungs, skin, and small intestines were collected postmortem for histological examination. No features of cytotoxicity [22] were evident in these tissues, in either control or peptide-treated groups. Renal and hepatic serum biochemistry parameters were within physiological limits [23] for all treatment groups (Figure 2(b)).

\subsection{Systemically Delivered StVOrth-2 Inhibits Osteosarcoma} Tumour Growth. Orthotopic osteosarcoma in this murine model was treated with systemically delivered StVOrth-2 and StVOrth-3. Microosmotic pumps, continuously delivering StVOrth-2 and StVOrth-3 at daily doses of $50 \mu \mathrm{g} / \mathrm{kg}$ and $500 \mu \mathrm{g} / \mathrm{kg}$, were implanted in the peritoneal cavities of mice at day 20 of the study. Tumours were well established at this time with an average tumour volume of $22.5 \mathrm{~mm}^{3}$ $( \pm 1.7$ SEM).

Sustained delivery of StVOrth-2 at both doses caused a significant reduction in primary tumour volume at the study endpoint. $50 \mu \mathrm{g} / \mathrm{kg}$ and $500 \mu \mathrm{g} / \mathrm{kg}$ StVOrth-2 caused $49.7 \%$ and $50.9 \%$ reductions in tumour volume, respectively, at day $34(P<0.01$, two-way ANOVA with Bonferroni multiple comparisons test) (Figures 3(a) and 3(b)). A significant effect 


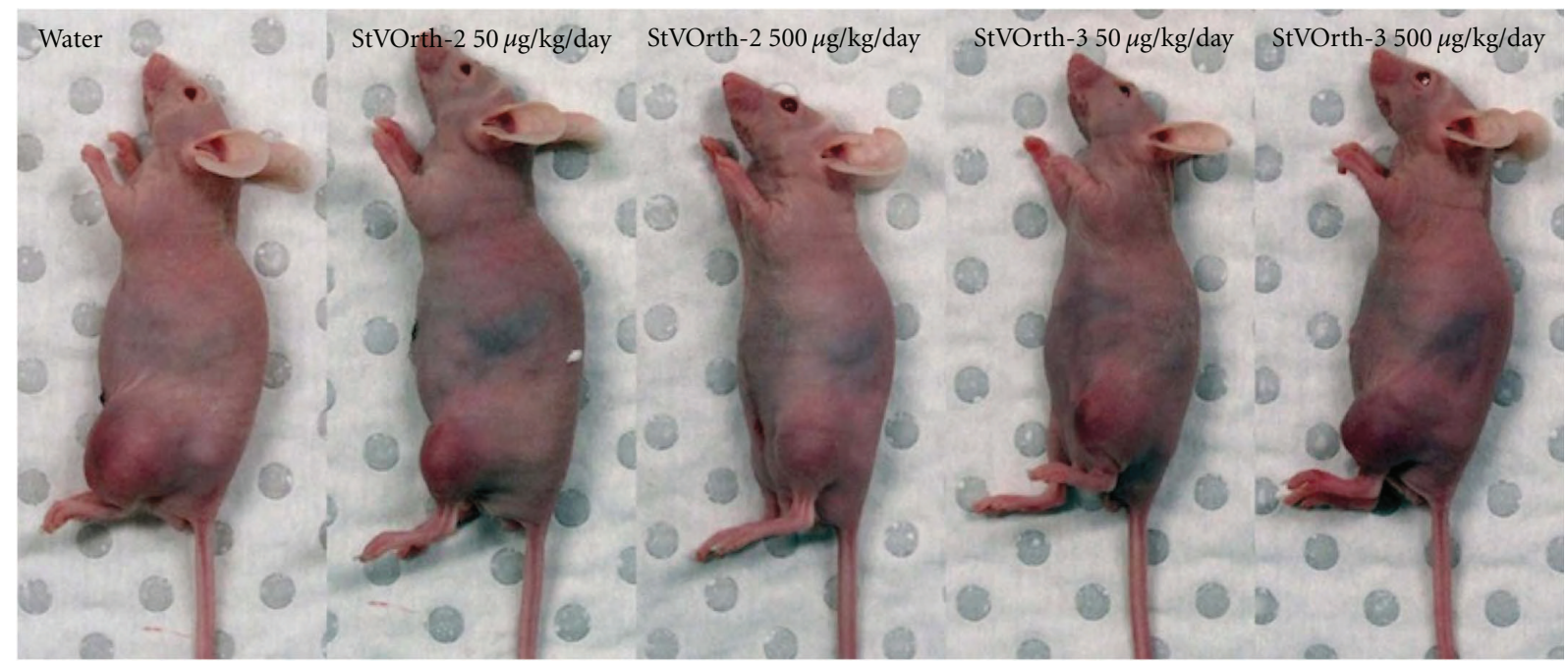

(a)

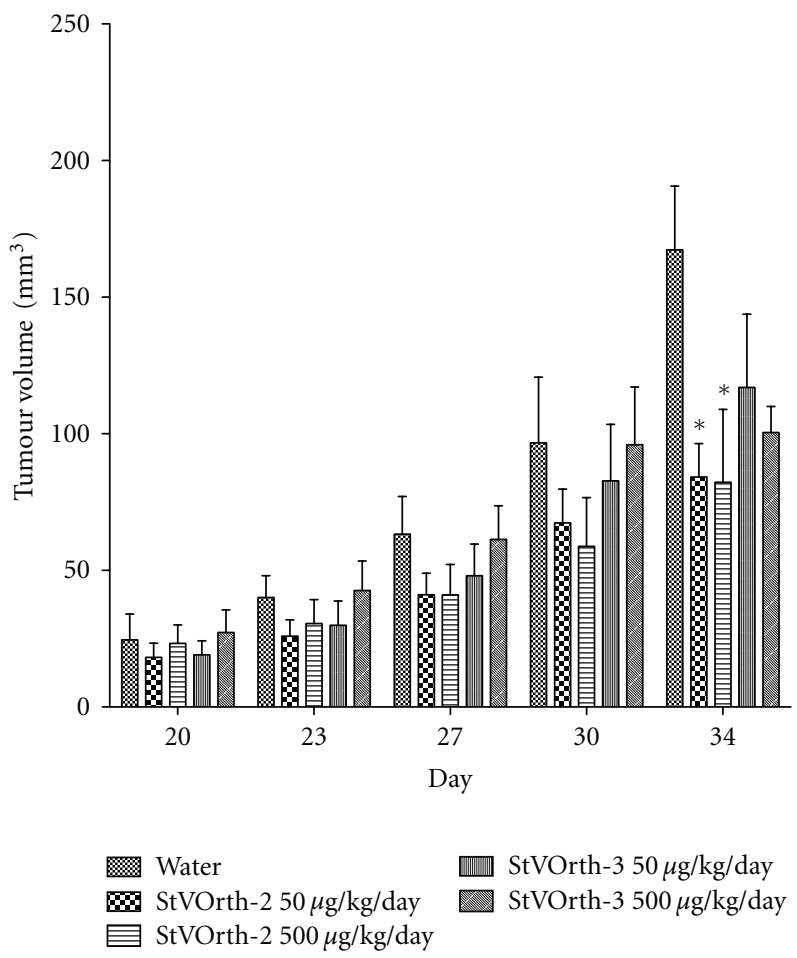

(b)

Figure 3: Systemically delivered StVOrth-2 inhibits growth of orthotopic osteosarcoma. (a) Photomicrographs of mice showing left tumourbearing hindlimbs. (b) Tumour volumes recorded at days $20,23,27,30$, and 34 after SaOS-2 intratibial injection $\left( \pm\right.$ SEM). ${ }^{*} P<0.01$, two-way ANOVA.

was not seen before the day 34 time point, and there was no statistical difference between the two doses of StVOrth-2.

By comparison, the therapeutic effect of StVOrth-2 on primary tumour volume was unable to be replicated by systemic StVOrth-3 therapy. Despite achieving 30.1\% and $40.0 \%$ reductions in primary tumour volume at day 34 with $50 \mu \mathrm{g} / \mathrm{kg}$ and $500 \mu \mathrm{g} / \mathrm{kg}$ StVOrth-3, respectively, these results did not achieve statistical significance (two-way ANOVA) (Figures 3(a) and 3(b)).
3.3. Tumour Invasion, Necrosis, and Apoptosis. Plain radiographs obtained after disarticulation of tumour-bearing limbs showed extensive soft tissue invasion and osteolysis for all animals (Figure 4). Invasion of surrounding structures, tumour necrosis, and apoptosis were assessed histologically. Treatment with either StVOrth-2 or StVOrth-3, at both $50 \mu \mathrm{g} / \mathrm{kg}$ and $500 \mu \mathrm{g} / \mathrm{kg}$ doses, did not affect orthotopic tumour invasion of the surrounding structures. All tumourbearing animals showed invasion of skeletal muscles and 

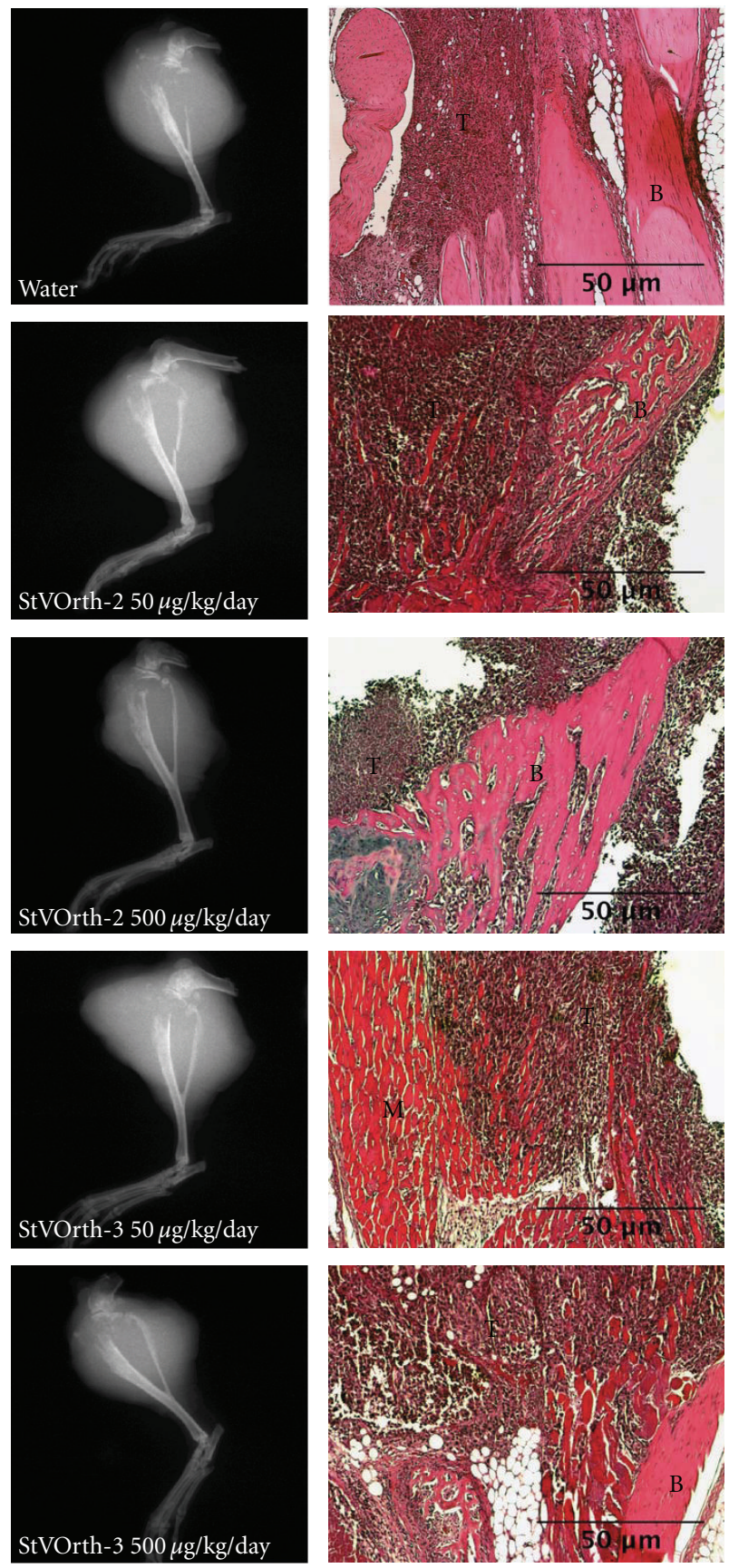

Figure 4: Tumour invasion. Plain radiographs (left) of tumourbearing limbs show extensive osteolysis of proximal tibiae and soft tissue extension for all treatment groups. Haematoxylin and eosinstained sections of orthotopic tumour (right) show tumour cells (T) invading bone (B) and skeletal muscle (M).

osteolysis on both sides of the adjacent joint. Tumour cells were clearly seen breaching the epiphyseal cartilage (Figure 4).

Treatment with StVOrth-2 or StVOrth-3 did not affect the degree of tumour necrosis. Per cent tumour necrosis was statistically equivalent for both StVOrth-2 and StVOrth-3 at either dose (one-way ANOVA). Similarly, treatment with
StVOrth-2 or StVOrth-3 did not appear to affect tumour cell apoptosis. The percentage of TUNEL-positive staining tumour was found to be statistically equivalent across treatment groups (one-way ANOVA) (Table 1).

\subsection{StVOrth-3 Restricts the Burden of Pulmonary Metastatic} Disease. This SaOS-2 orthotopic model of osteosarcoma gives rise to spontaneous pulmonary metastases, and the burden of metastatic disease was assessed histologically postmortem. Haematoxylin- and eosin-stained sections of lung tissue were examined under 20x objective. The numbers of micrometastases per lung section were enumerated, and treatment with StVOrth-2 or StVOrth-3, at both $50 \mu \mathrm{g} / \mathrm{kg}$ and $500 \mu \mathrm{g} / \mathrm{kg}$ doses, had no effect on the number of lesions observed (one-way ANOVA) (Figures 5(a) and 5(b)).

Treatment with StVOrth-3 did, however, cause a significant reduction in the size of micrometastatic lesions. The mean cross-sectional area of a micrometastasis was $0.19 \mathrm{~mm}^{2}$ and $0.29 \mathrm{~mm}^{2}$ for $50 \mu \mathrm{g} / \mathrm{kg}$ and $500 \mu \mathrm{g} / \mathrm{kg}$ StVOrth-3. This represented a $79 \%$ and $68.2 \%$ reduction in area compared to animals that received sterile water as control $(P<0.05$, oneway ANOVA analysis with Bonferroni multiple comparisons test). Treatment with $50 \mu \mathrm{g} / \mathrm{kg}$ and $500 \mu \mathrm{g} / \mathrm{kg}$ StVOrth-2 resulted in mean cross-sectional areas of $0.68 \mathrm{~mm}^{2}$ and $1.02 \mathrm{~mm}^{2}$ respectively. These results, however, were not statistically significant (Figure 5(c)).

\section{Discussion}

Ek et al. [7] evaluated four PEDF-derived peptides based on the known functional epitopes of the PEDF glycoprotein. StVOrth-2 (residues 78-102) predominantly restricted SaOS-2 osteosarcoma cell proliferation and StVOrth-3 (residues 90-114) increased adhesion to collagen I. Both peptides restricted growth of primary osteosarcoma and the development of pulmonary metastases in vivo [7]. In this study we aimed to assess systemically delivered StVOrth-2 and StVOrth-3 as treatments for established osteosarcoma (both primary and secondary disease) and to evaluate the therapeutic safety of these peptides. We utilised the same orthotopic murine model of osteosarcoma described by Ek et al. [7] and delivered the StVOrth-2 and StVOrth-3 peptides systemically via an intraperitoneal microosmotic pump. Treatment was delayed until after the macroscopic appearance of primary osteosarcoma. Using this optimised model, differential effects on tumour growth and metastasis were demonstrated for StVOrth-2 and StVOrth-3, respectively.

Systemic delivery of StVOrth-2 restricted the volume of primary osteosarcoma. We observed $49.7 \%$ and $50.9 \%$ reductions in tumour volume with $50 \mu \mathrm{g} / \mathrm{kg}$ and $500 \mu \mathrm{g} / \mathrm{kg}$ StVOrth-2 treatments, respectively. Ek et al. [7] showed a reduction of more then $30 \%$ when these peptides were administered prior to SaOS-2 inoculation. It is interesting that StVOrth-2 caused greater inhibition of tumour growth than StVOrth-3. Ek et al. [7] showed that StVOrth-2 had a significant antiproliferative effect on SaOS-2 cells in vitro, while StVOrth-3 did not exhibit this effect. The 
TABLE 1: Tumour per cent necrosis and apoptosis as determined on haematoxylin and eosin-stained and TUNEL-stained sections of orthotopic tumour, respectively.

\begin{tabular}{lccccc}
\hline & Water & StVOrth-2 $50 \mu \mathrm{g} / \mathrm{kg} /$ day & StVOrth-2 $500 \mu \mathrm{g} / \mathrm{kg} / \mathrm{day}$ & StVOrth-3 50 $\mu \mathrm{g} / \mathrm{kg} / \mathrm{day}$ & StVOrth-3 500 $\mu \mathrm{g} / \mathrm{kg} / \mathrm{day}$ \\
\hline \multirow{2}{*}{$\%$ Necrosis } & $28.2 \%$ & $31.3 \%$ & $8.9 \%$ & $46.6 \%$ & $33.5 \%$ \\
& $( \pm 8.01 \mathrm{SEM})$ & $( \pm 11.85 \mathrm{SEM}) P>0.05$ & $( \pm 2.952 \mathrm{SEM}) P>0.05$ & $( \pm 10.04 \mathrm{SEM}) P>0.05$ & $( \pm 4.071 \mathrm{SEM}) P>0.05$ \\
\multirow{2}{*}{$\%$ Apoptosis $P$} & $20.2 \%$ & $15.9 \%$ & $3.6 \%$ & $39.4 \%$ & $41.3 \%$ \\
& $( \pm 2.22 \mathrm{SEM})$ & $( \pm 1.87 \mathrm{SEM}) P>0.05$ & $( \pm 0.56 \mathrm{SEM}) P>0.05$ & $( \pm 13.89 \mathrm{SEM}) P>0.05$ & $( \pm 10.12 \mathrm{SEM}) P>0.05$ \\
\hline
\end{tabular}

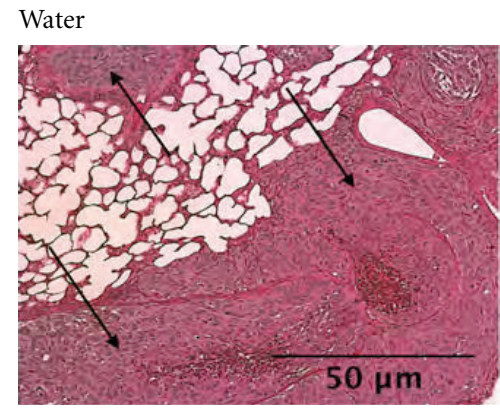

StVOrth-3 $50 \mu \mathrm{g} / \mathrm{kg} /$ day

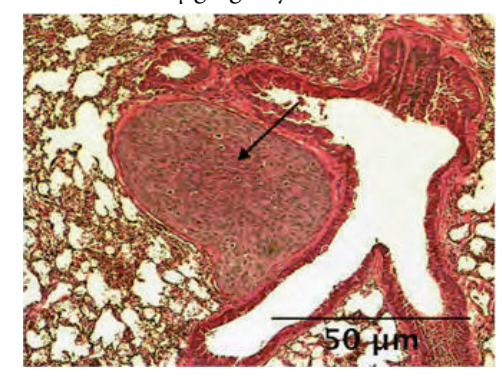

StVOrth-2 $50 \mu \mathrm{g} / \mathrm{kg} /$ day

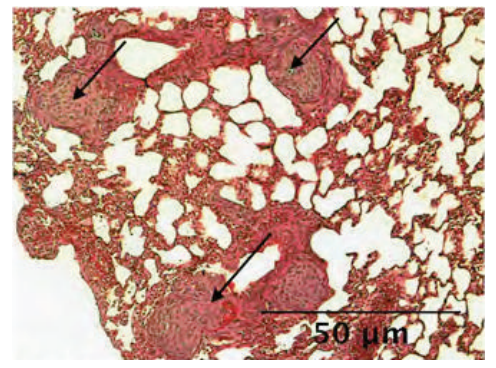

StVOrth-2 $500 \mu \mathrm{g} / \mathrm{kg} /$ day

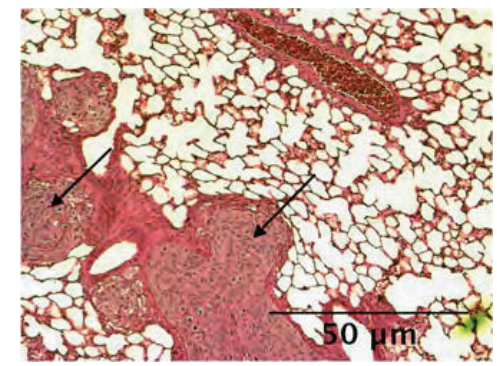

StVOrth-3 $500 \mu \mathrm{g} / \mathrm{kg} /$ day

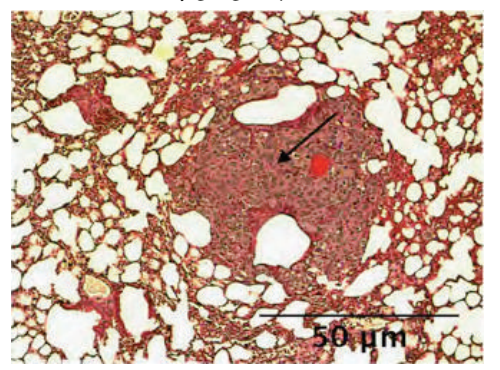

(a)

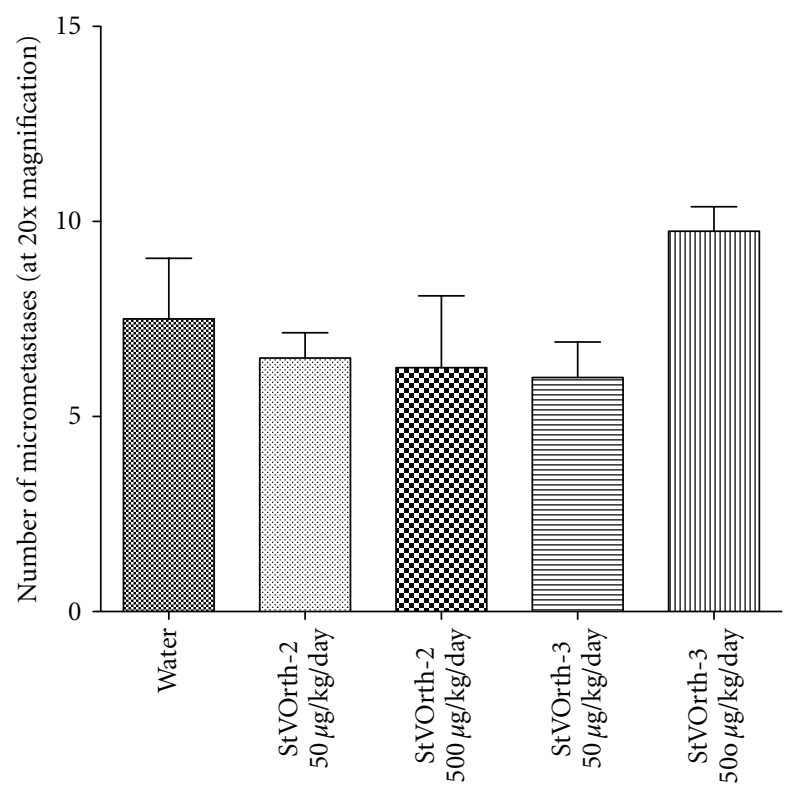

(b)

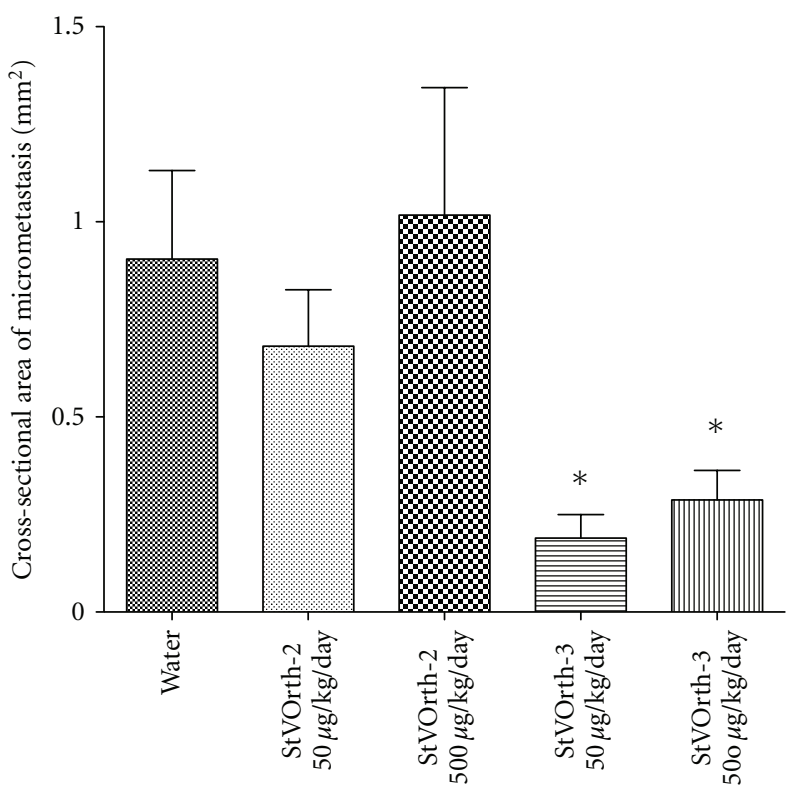

(c)

Figure 5: Systemically delivered StVOrth-3 inhibits progression of pulmonary metastatic disease. (a) Haematoxylin- and eosin-stained sections of lung tissue show micrometastases (arrows) disrupting the native alveolar architecture. Metastatic lesions were predominately subpleural and around larger airways. (b) Number of pulmonary micrometastases per lung section at 20x magnification ( \pm SEM). (c) Crosssectional areas of pulmonary micrometastases $( \pm$ SEM $) .{ }^{*} P<0.05$, one-way ANOVA. 
molecular mechanisms exploited by StVOrth-2 to achieve its antitumour effect are yet to be characterised.

Treatment with StVOrth-2 and StVOrth-3 did not appear to affect local tumour invasion, tumour necrosis, or apoptosis. Tumour cells were observed invading the soft tissues and destroying the local bony architecture in all animals, independent of treatment type. Although groups receiving StVOrth-2 and StVOrth-3 were unable to be statistically differentiated based on tumour cell necrosis and apoptosis, it was evident that the percentages of tumour necrosis and apoptosis were proportional to tumour volume. This SaOS-2 orthotopic model of osteosarcoma gives rise to large and rapidly growing tumours. By delaying treatment until tumours were palpable, it is possible that tumour energy requirements exceeded capacity of the vascular supply, leading to spontaneous necrosis and apoptosis of the tumour core. Such areas of necrosis and potential apoptosis are commonly observed in clinical tumours at our centre (personal observation).

Ek et al. [7] showed that both StVOrth-2 and StVOrth-3 dramatically restricted spontaneous pulmonary metastases, despite exhibiting differential effects on cell proliferation and collagen I invasion in vitro. The authors hypothesized that distinct antitumour pathways may explain these results. With this is mind, the role of these peptides in the metastatic process was again investigated. Pulmonary micrometastases were quantified under low-power microscopy, rather than the postmortem macroscopic method utilised by Ek et al. [7]. Cross-sectional areas of metastatic lesions were quantified to estimate the burden of pulmonary disease. StVOrth-3 showed a dramatic $79 \%$ reduction in pulmonary burden of disease, an effect consistent with the observation that StVOrth-3 predominantly restricts adhesion to collagen I [7]. StVOrth-3 may critically inhibit the metastatic cascade via this mechanism; however, one cannot rule out a direct effect on pulmonary lesions via systemic delivery. The method used here for evaluating metastatic lesions is an improvement upon that used by Ek et al. [7].

The final aim of this study was to perform a preliminary evaluation of therapeutic safety. Ek et al. [7] showed that both StVOrth-2 and StVOrth-3 possess a moderate ability to inhibit VEGF expression by SaOS-2 cells. For antiangiogenic agents to be of clinical use, it is critical that these effects are not achieved at the expense of homeostatic processes such as wound healing and inflammation. Serum analysis and histology of harvested viscera did not show any evidence of treatment-related toxicity. All animals appeared to be healthy and exhibited normal grazing and behaviour for the duration of the study. We also did not note any gross differences in surgical wound healing between the treatment groups.

We also clarify that both StVOrth-2 and StVOrth-3 were nonimmunogenic, despite their length (25-mer). There are two direct items that need to be highlighted: (i) both peptides were effective in vivo when administered systemically (despite any potential immunogenicity), and (ii) we did not notice any inflammation- or immunostimulation-mediated exudates around the region of the pump outlet. If the peptides were immunogenic, this would be clearly visible in these mice, as we have noticed this before with other anticancer agents (Dass, unpublished data).

StVOrth-2 has been shown to be most effective at inhibiting primary tumour growth while StVOrth-3 predominantly restricts progression of pulmonary metastases. The molecular mechanisms utilised by these peptides are yet to be fully studied and elucidated. Mechanistic studies have focused on the parent PEDF glycoprotein that interacts with regulatory pathways that induce apoptosis of both endothelial and tumour cells [24]. In vitro and in vivo studies have implicated the Fas-FasL death receptor pathway [25-27], Bcl-2 family proteins [28], caspases, and c-Flip [29]. The current study did not look at molecular markers in vivo, apart from the blood analyses for creatinine, alanine transaminase and aspartate transferase. In vitro though, we have previously examined several biological and molecular features of PEDFbased peptide treatment of SaOS-2 cells [7]. In that earlier study, StVOrth-2 was the most potent inhibitor of SaOS2 osteosarcoma cell proliferation, while StVOrth-3 dramatically promoted SaOS-2 adhesion to collagen I. StVOrth-2 and -3 induced osteoblastic differentiation, while StVOrth3 and StVOrth- 4 reduced VEGF expression in SaOS-2 cells. The current set of in vivo results, from a focussed pharmacodynamic study, reflect quite closely what we expected from the earlier in vitro study.

Peptides have a number of advantages as targeted therapies, being neither genotoxic nor genotype specific [30]. Peptides have excellent tissue penetration and bind with high affinity and specificity to therapeutic targets [31-33]. In this study we demonstrated pronounced differences in action between the two peptides and this begs the question as to precisely which are the aminoacid sequences essential for these effects. StVOrth-2 consisted of ${ }_{78}$ LSPLS VATAL SALSL GAEQR TESII ${ }_{102}$, and StVOrth-3 consisted of ${ }_{90}$ LSLGA EQRTE SIIHR ALYYD LISSP ${ }_{114}$. The overlapping ${ }_{90} \mathrm{LSL}$ GAEQR TESII $_{102}$ sequence may in fact be nonessential for the described effects. Further studies utilising the shortened, nonoverlapping sequences of StVOrth-2 and StVOrth-3 are the next logical step. Beyond this, development of a hybrid version of StVOrth-2 and StVOrth-3 peptides may then be possible, leading potentially to a compound that possesses antitumorigenic and antimetastatic properties superior to that of the parent PEDF glycoprotein and hopefully of the individual peptides used in isolation.

Multiple functional epitopes for PEDF have been identified, as well as several distinct PEDF receptors [34, 35]. Filleur at al. [6] first characterised 34-mer (residues 24-57) and 44-mer (residues 58-101) PEDF-derived peptides that conferred antiangiogenic and neurotrophic activity, respectively. Additionally, another sequence, ERT (residues 79-94), showed both antiangiogenic and differentiation activity. Mirochnik et al. [26] derived three different shorter peptides from the 34-mer PEDF-based peptide [6] and demonstrated that a 18 -mer peptide was in fact able to inhibit angiogenesis and the growth of prostate cancer in mice. Amaral and Becerra [36] showed that the 34 mer peptide [6] suppresses choroidal neovascularization following subconjunctival administration. The interactions between these various identified epitopes and receptors 
are likely to initiate divergent signalling pathways for the different cellular effects of PEDF. We did not identify which PEDF receptor the peptides were interacting with in this study. There is also the possibility that the peptides may be interacting with other cell surface ligands given that the quaternary structure of the peptides is expected to be very different from the full length PEDF protein.

\section{Conclusions}

This study provides a number of insights into the mechanisms of the antiosteosarcoma activity of PEDF and supports the use of PEDF-derived peptides as therapeutic agents. StVOrth-2 (residues 78-102) primarily restricts growth of primary osteosarcoma, while StVOrth-3 (residues 90-114) restricts pulmonary metastatic disease. These findings are particularly significant given that the orthotopic model of osteosarcoma gives rise to large and rapidly growing tumours, and that these peptides were administered at a stage of advanced disease.

\section{Acknowledgments}

This work was funded by the Liddy Shriver Sarcoma Initiative. Dr. M. L. Broadhead is supported by a Medical Postgraduate Scholarship, awarded by the National Health and Medical Research Council (NHMRC, Australia). The authors thank the Australian Orthopaedic Association (AOA) and the Royal Australasian College of Surgeons (RACS) for their ongoing support.

\section{References}

[1] S. P. Becerra, "Focus on molecules: pigment epitheliumderived factor (PEDF)," Experimental Eye Research, vol. 82, no. 5, pp. 739-740, 2006.

[2] J. Tombran-Tink, G. G. Chader, and L. V. Johnson, "PEDF: a pigment epithelium-derived factor with potent neuronal differentiative activity," Experimental Eye Research, vol. 53, no. 3, pp. 411-414, 1991.

[3] J. Tombran-Tink and C. J. Barnstable, "Therapeutic prospects for PEDF: more than a promising angiogenesis inhibitor," Trends in Molecular Medicine, vol. 9, no. 6, pp. 244-250, 2003.

[4] D. W. Dawson, O. V. Volpert, P. Gillis et al., "Pigment epithelium-derived factor: a potent inhibitor of angiogenesis," Science, vol. 285, no. 5425, pp. 245-248, 1999.

[5] M. L. Broadhead, C. R. Dass, and P. F. Choong, "In vitro and in vivo biological activity of PEDF against a range of tumors," Expert opinion on therapeutic targets, vol. 13, no. 12, pp. 1429$1438,2009$.

[6] S. Filleur, K. Volz, T. Nelius et al., "Two functional epitopes of pigment epithelial-derived factor block angiogenesis and induce differentiation in prostate cancer," Cancer Research, vol. 65, no. 12, pp. 5144-5152, 2005.

[7] E. T. H. Ek, C. R. Dass, K. G. Contreras, and P. F. M. Choong, "PEDF-derived synthetic peptides exhibit antitumor activity in an orthotopic model of human osteosarcoma," Journal of Orthopaedic Research, vol. 25, no. 12, pp. 1671-1680, 2007.

[8] M. L. Broadhead, J. C. Clark, P. F. Choong, and C. R. Dass, "Editorial: making gene therapy for osteosarcoma a reality,"
Expert Review of Anticancer Therapy, vol. 10, no. 4, pp. 477480, 2010.

[9] C. R. Dass, E. T. Ek, K. G. Contreras, and P. F. Choong, "A novel orthotopic murine model provides insights into cellular and molecular characteristics contributing to human osteosarcoma," Clinical and Experimental Metastasis, vol. 23, no. 7-8, pp. 367-380, 2006.

[10] K. Matsumoto, H. Ishikawa, D. Nishimura, K. Hamasaki, K. Nakao, and K. Eguchi, "Antiangiogenic property of pigment epithelium-derived factor in hepatocellular carcinoma," Hepatology, vol. 40, no. 1, pp. 252-259, 2004.

[11] K. Nakamura, S. I. Yamagishi, H. Adachi, Y. Kurita-Nakamura, T. Matsui, and H. Inoue, "Serum levels of pigment epitheliumderived factor (PEDF) are positively associated with visceral adiposity in Japanese patients with type 2 diabetes," Diabetes/Metabolism Research and Reviews, vol. 25, no. 1, pp. 52$56,2009$.

[12] M. Sabater, J. M. Moreno-Navarrete, F. J. Ortega et al., "Circulating pigment epithelium-derived factor levels are associated with insulin resistance and decrease after weight loss," Journal of Clinical Endocrinology and Metabolism, vol. 95, no. 10, pp. 4720-4728, 2010.

[13] K. Sogawa, Y. Kodera, M. Satoh et al., "Increased serum levels of pigment epithelium-derived factor by excessive alcohol consumption-detection and identification by a three-step serum proteome analysis," Alcoholism: clinical and Experimental Research, vol. 35, no. 2, pp. 211-217, 2011.

[14] A. Wiercinska-Drapalo, J. Jaroszewicz, A. Parfieniuk, T. W. Lapinski, M. Rogalska, and D. Prokopowicz, "Pigment epithelium-derived factor in ulcerative colitis: possible relationship with disease activity," Regulatory Peptides, vol. 140, no. 1-2, pp. 1-4, 2007.

[15] S. Yang, Q. Li, L. Zhong et al., "Serum pigment epitheliumderived factor is elevated in women with polycystic ovary syndrome and correlates with insulin resistance," Journal of Clinical Endocrinology and Metabolism, vol. 96, no. 3, pp. 831836, 2011.

[16] V. Stellmach, S. E. Crawford, W. Zhou, and N. Bouck, "Prevention of ischemia-induced retinopathy by the natural ocular antiangiogenic agent pigment epithelium-derived factor," Proceedings of the National Academy of Sciences of the United States of America, vol. 98, no. 5, pp. 2593-2597, 2001.

[17] "Frequently asked questions," The Jackson Laboratory 2011.

[18] O. Kisker, C. M. Becker, D. Prox et al., "Continuous administration of endostatin by intraperitoneally implanted osmotic pump improves the efficacy and potency of therapy in a mouse xenograft tumor model," Cancer Research, vol. 61, no. 20, pp. 7669-7674, 2001.

[19] D. Kurosaka, K. Yoshida, J. Yasuda et al., "The effect of endostatin evaluated in an experimental animal model of collageninduced arthritis," Scandinavian Journal of Rheumatology, vol. 36, no. 6, pp. 434-441, 2007.

[20] R. G. Fahmy, C. R. Dass, L. Q. Sun, C. N. Chesterman, and L. M. Khachigian, "Transcription factor Egr-1 supports FGFdependent angiogenesis during neovascularization and tumor growth," Nature Medicine, vol. 9, no. 8, pp. 1026-1032, 2003.

[21] H. T. Ta, C. R. Dass, I. Larson, P. F. M. Choong, and D. E. Dunstan, "A chitosan hydrogel delivery system for osteosarcoma gene therapy with pigment epithelium-derived factor combined with chemotherapy," Biomaterials, vol. 30, no. 27, pp. 4815-4823, 2009.

[22] M. L. Tan, A. M. Friedhuber, D. E. Dunstan, P. F. M. Choong, and C. R. Dass, "The performance of doxorubicin encapsulated in chitosan-dextran sulphate microparticles in an 
osteosarcoma model," Biomaterials, vol. 31, no. 3, pp. 541-551, 2010.

[23] "Reference values for laboratory animals," Research Animal Resources, University of Minnesota, 2009.

[24] M. L. Broadhead, C. R. Dass, and P. F. M. Choong, "Cancer cell apoptotic pathways mediated by PEDF: prospects for therapy," Trends in Molecular Medicine, vol. 15, no. 10, pp. 461-467, 2009.

[25] R. Abe, T. Shimizu, S. I. Yamagishi et al., "Overexpression of pigment epithelium-derived factor decreases angiogenesis and inhibits the growth of human malignant melanoma cells in vivo," American Journal of Pathology, vol. 164, no. 4, pp. 12251232, 2004.

[26] Y. Mirochnik, A. Aurora, F. T. Schulze-Hoepfner et al., "Short pigment epithelial-derived factor-derived peptide inhibits angiogenesis and tumor growth," Clinical Cancer Research, vol. 15, no. 5, pp. 1655-1663, 2009.

[27] O. V. Volpert, T. Zaichuk, W. Zhou et al., "Inducer-stimulated Fas targets activated endothelium for destruction by antiangiogenic thrombospondin-1 and pigment epitheliumderived factor," Nature Medicine, vol. 8, no. 4, pp. 349-357, 2002.

[28] T. Zhang, M. Guan, C. Xu, Y. Chen, and Y. Lu, "Pigment epithelium-derived factor inhibits glioma cell growth in vitro and in vivo," Life Sciences, vol. 81, no. 16, pp. 1256-1263, 2007.

[29] T. A. Zaichuk, E. H. Shroff, R. Emmanuel, S. Filleur, T. Nelius, and O. V. Volpert, "Nuclear factor of activated T cells balances angiogenesis activation and inhibition," Journal of Experimental Medicine, vol. 199, no. 11, pp. 1513-1522, 2004.

[30] S. K. Bhutia and T. K. Maiti, "Targeting tumors with peptides from natural sources," Trends in Biotechnology, vol. 26, no. 4, pp. 210-217, 2008.

[31] J. S. Mader and D. W. Hoskin, "Cationic antimicrobial peptides as novel cytotoxic agents for cancer treatment," Expert Opinion on Investigational Drugs, vol. 15, no. 8, pp. 933-946, 2006.

[32] C. Rüegg, M. Hasmim, F. J. Lejeune, and G. C. Alghisi, "Antiangiogenic peptides and proteins: from experimental tools to clinical drugs," Biochimica et Biophysica Acta, vol. 1765, no. 2, pp. 155-177, 2006.

[33] Y. L. Janin, "Peptides with anticancer use or potential," Amino Acids, vol. 25, no. 1, pp. 1-40, 2003.

[34] L. Notari, V. Baladron, J. D. Aroca-Aguilar et al., "Identification of a lipase-linked cell membrane receptor for pigment epithelium-derived factor," Journal of Biological Chemistry, vol. 281, no. 49, pp. 38022-38037, 2006.

[35] A. Bernard, J. Gao-Li, C. A. Franco, T. Bouceba, A. Huet, and $\mathrm{Z}$. Li, "Laminin receptor involvement in the anti-angiogenic activity of pigment epithelium-derived factor," Journal of Biological Chemistry, vol. 284, no. 16, pp. 10480-10490, 2009.

[36] J. Amaral and S. P. Becerra, "Effects of human recombinant PEDF protein and PEDF-derived peptide 34-mer on choroidal neovascularization," Investigative ophthalmology \& visual science, vol. 51, no. 3, pp. 1318-1326, 2010. 

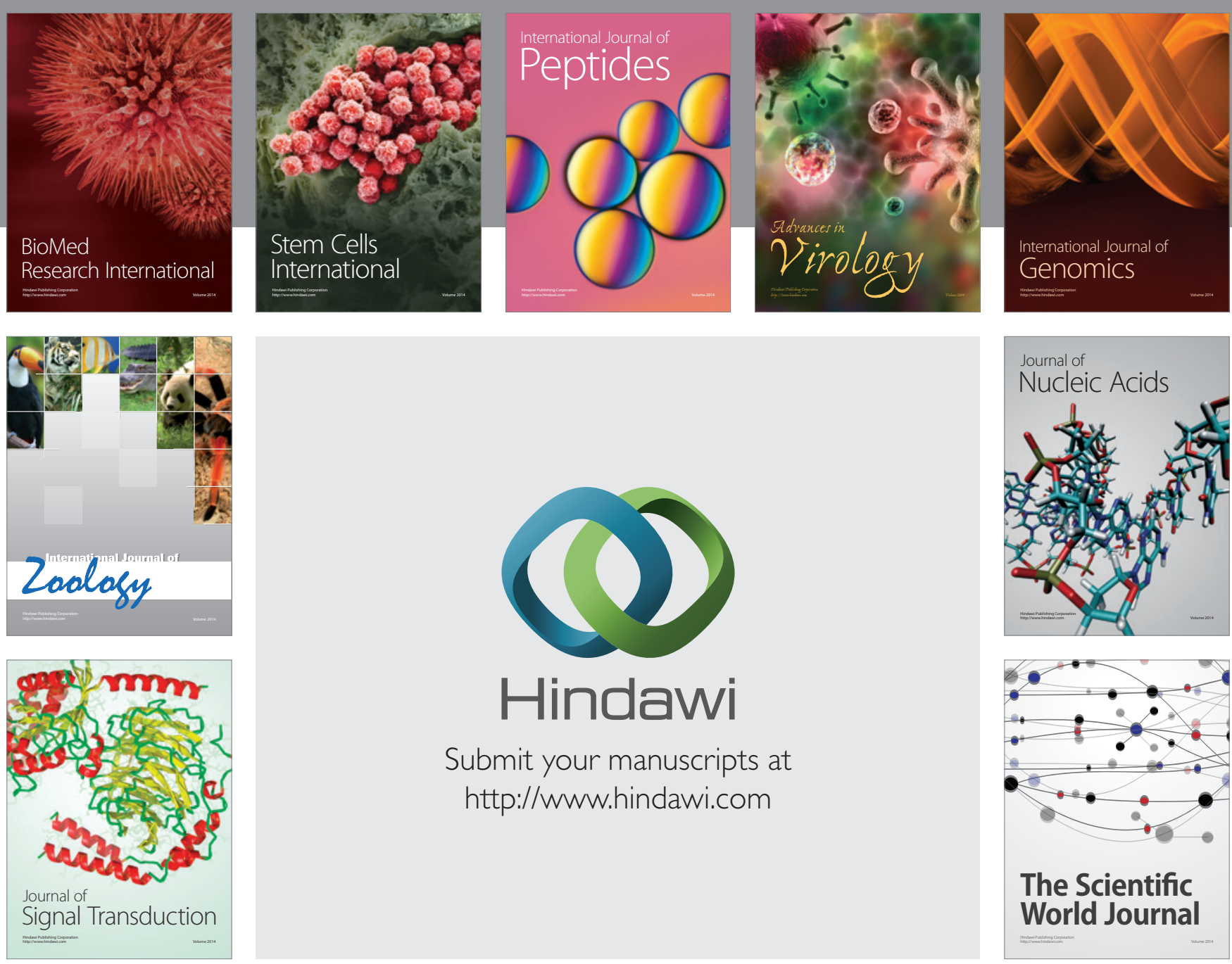

Submit your manuscripts at

http://www.hindawi.com
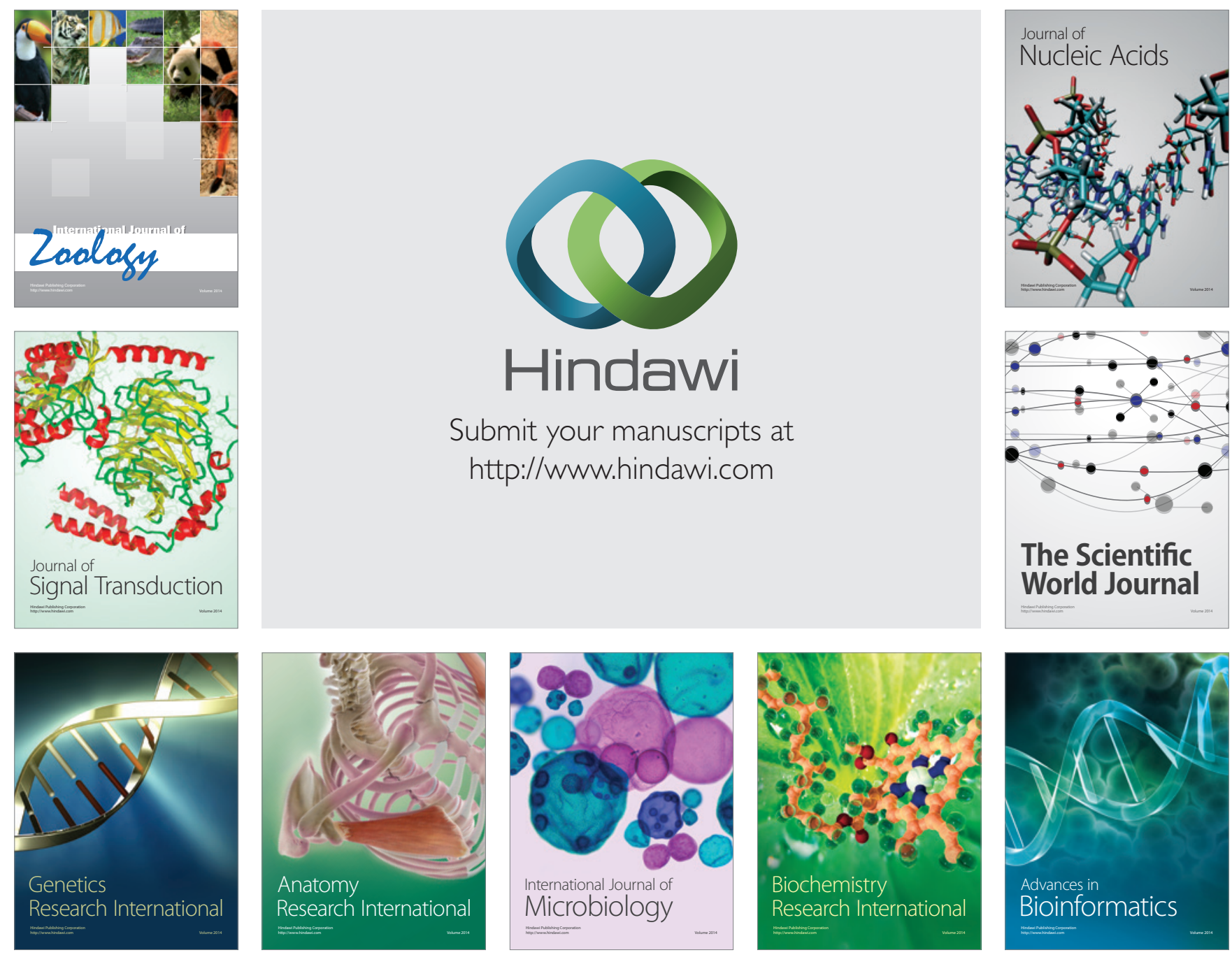

The Scientific World Journal
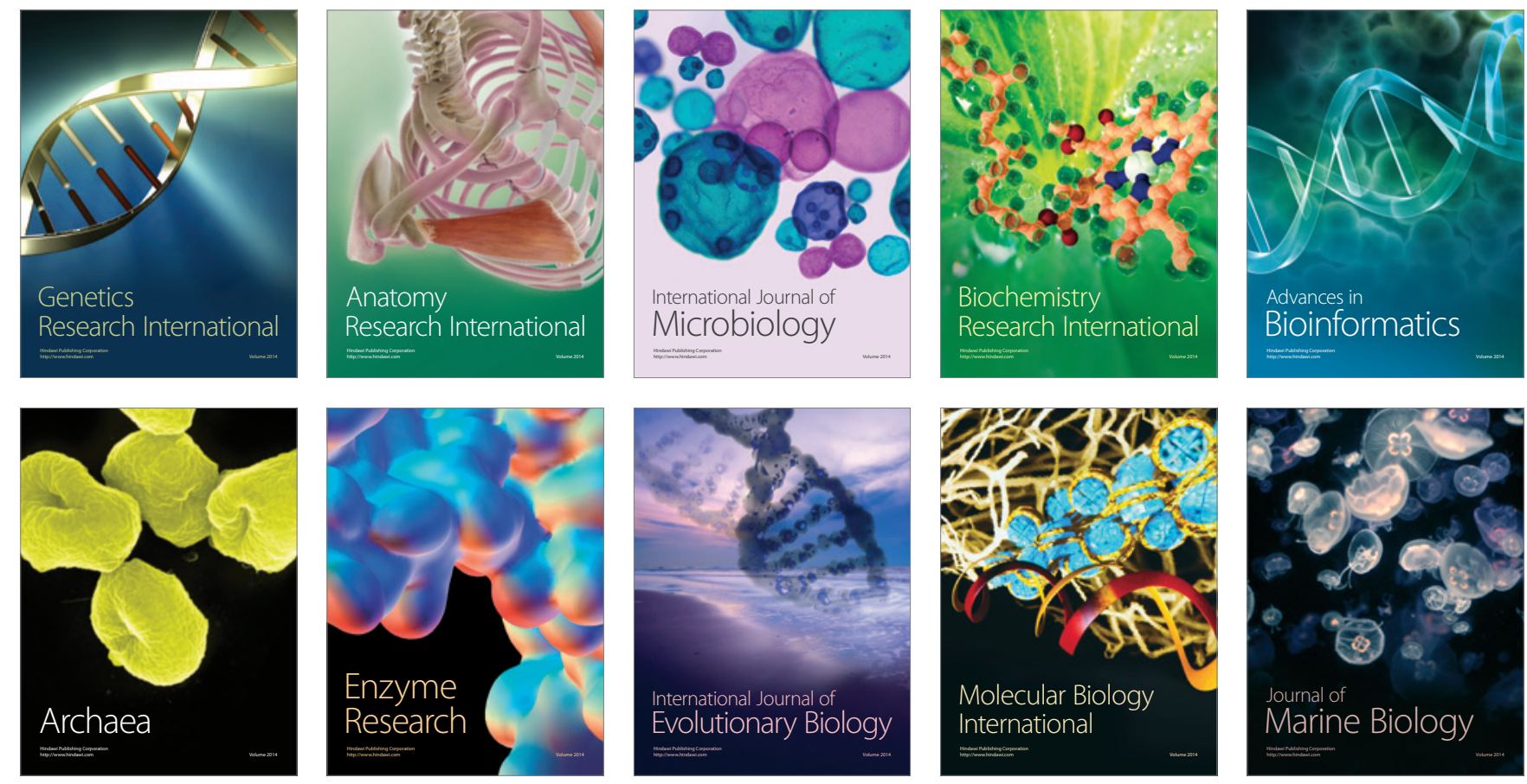